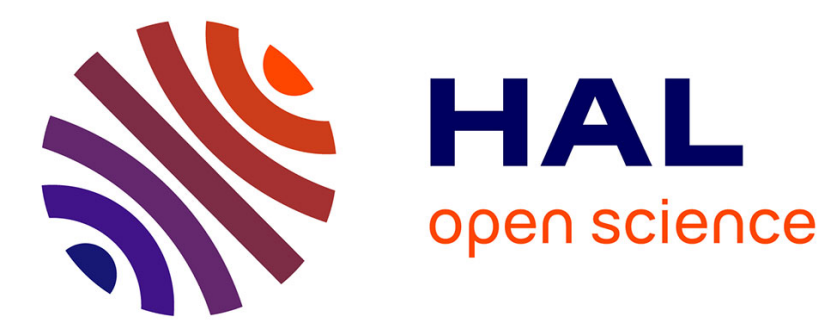

\title{
Multi-Class Leveraged $k$-NN for Image Classification
}

Paolo Piro, Richard Nock, Frank Nielsen, Michel Barlaud

\section{To cite this version:}

Paolo Piro, Richard Nock, Frank Nielsen, Michel Barlaud. Multi-Class Leveraged $k$-NN for Image Classification. Proceedings of the 10th Asian Conference on Computer Vision, ACCV 2010, November 8-12, 2010, Queenstown, New Zealand, 2010, Queenstown, New Zealand. hal-00664606

\section{HAL Id: hal-00664606 https://hal.inria.fr/hal-00664606}

Submitted on 31 Jan 2012

HAL is a multi-disciplinary open access archive for the deposit and dissemination of scientific research documents, whether they are published or not. The documents may come from teaching and research institutions in France or abroad, or from public or private research centers.
L'archive ouverte pluridisciplinaire HAL, est destinée au dépôt et à la diffusion de documents scientifiques de niveau recherche, publiés ou non, émanant des établissements d'enseignement et de recherche français ou étrangers, des laboratoires publics ou privés. 


\title{
Multi-Class Leveraged $k$-NN for Image Classification
}

\author{
Paolo Piro ${ }^{1}$, Richard Nock ${ }^{2}$, Frank Nielsen ${ }^{3}$, and Michel Barlaud ${ }^{1}$ \\ 1 University of Nice-Sophia Antipolis / CNRS, France \\ 2 CEREGMIA, University of Antilles-Guyane, France \\ 3 Sony CSL / LIX, Ecole Polytechnique, France
}

\begin{abstract}
The $k$-nearest neighbors ( $k$-NN) classification rule is still an essential tool for computer vision applications, such as scene recognition. However, $k$-NN still features some major drawbacks, which mainly reside in the uniform voting among the nearest prototypes in the feature space.

In this paper, we propose a new method that is able to learn the "relevance" of prototypes, thus classifying test data using a weighted $k$-NN rule. In particular, our algorithm, called Multi-class Leveraged $k$-nearest neighbor (MLNN), learns the prototype weights in a boosting framework, by minimizing a surrogate exponential risk over training data. We propose two main contributions for improving computational speed and accuracy. On the one hand, we implement learning in an inherently multiclass way, thus providing significant computation time reduction over one-versus-all approaches. Furthermore, the leveraging weights enable effective data selection, thus reducing the cost of $k$-NN search at classification time. On the other hand, we propose a kernel generalization of our approach to take into account real-valued similarities between data in the feature space, thus enabling more accurate estimation of the local class density.

We tested MLNN on three datasets of natural images. Results show that MLNN significantly outperforms classic $k$-NN and weighted $k$-NN voting. Furthermore, using an adaptive Gaussian kernel provides significant performance improvement. Finally, the best results are obtained when using MLNN with an appropriate learned metric distance.
\end{abstract}

\section{Introduction}

In this paper, we address the task of image categorization. This task aims at automatically classifying images into a predefined set of scene categories, like the natural scenes represented in Fig. 1. (See Sec. 3.1 for a detailed description of the databases we used in our experiments). Despite lots of works, much remains to be done to challenge human level performances, not only because there is a huge number of natural categories that should be considered in general. In fact, images carry only parts of the information that is used by humans to categorize, and parts of the information available from images may be highly misleading: for example, natural image categories may exhibit high intra-class variability (i.e., visually different images may belong to the same category) and low inter-class variability (i.e., distinct categories may contain images that are visually similar). 


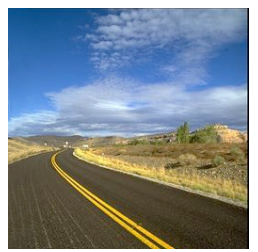

highway

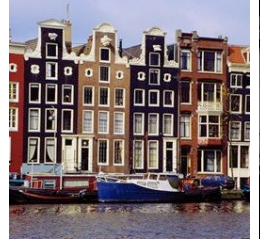

inside city

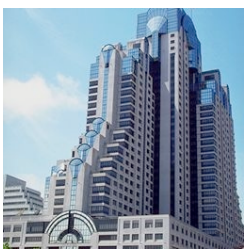

tall building

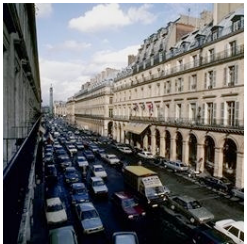

street

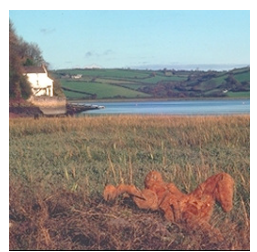

open country

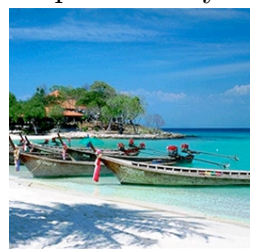

coast

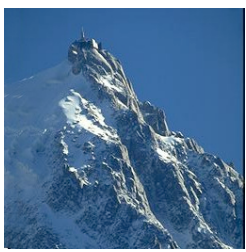

mountain

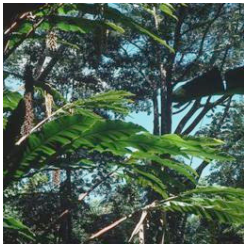

forest

Fig. 1. The first dataset we used in our experiments consists of 8 categories of natural scenes [1].

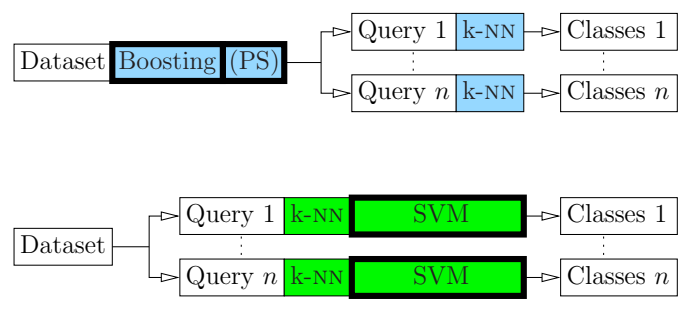

Fig. 2. Optimizing $k$-NN via MLNN (up, blue) and SVM-KNN [2] (down, green). MLNN uses a boosting algorithm before being presented any query, while SVM-KNN learns support vectors after each query is presented. Bold rectangles indicate induction steps ( $\mathrm{PS}=$ prototype selection; see text for details).

For the purpose of automatic classification of images, the $k$-NN classification has shown to be very effective [3]. Its use is supported by a wide spectrum of arguments, ranging from the field of philosophy to that of mathematics [2]. The simplicity of the method - use the labeled neighbor(s) of a query to predict its class - makes it a good candidate for further improvements, desirable in part because of statistical and computational drawbacks [2]. So far, the literature has favoured two main ways to cope with these issues: improve classification accuracy by means of local classifiers $[2,4,5,6]$, or filter out ill-defined examples [7], with intermediate approaches [8]. Many of these algorithms can be viewed as primers to improve the (continuous) estimation of class membership probabilities [9], but none has completely succeeded in this task. This problem has been reformulated by Marin et al. [10] as a strong advocacy for the formal transposition of boosting to $k$-NN classification. This issue is challenging as $k$-NN rules are indeed not induced, whereas formal boosting algorithms combine $t w o$ induction steps, 
inducing so-called strong classifiers by combining weak classifiers (also induced). Previously, Athitsos and Sclaroff [11] had already proposed an approach to bring the boosting principle into the $k$-NN classification framework. However, their method consisted in "boosting" the distance measure, i.e., learning a combination of metric distances that could improve the generalization of classifier. Furthermore, their classification framework was not intrinsically multiclass, as they formulated the problem as binary learning on random triplets of training data.

In this paper, we tackle the issue of integrating $k$-NN in a boosting framework from a different perspective. In particular, our algorithm, called MLNN, induces a multiclass leveraged $k$-nearest neighbor rule that generalizes the uniform $k$-NN rule, using the examples directly as weak hypotheses (that we also call prototypes). Our MLNN method does not need to learn a distance function, as it directly operates on the top of $k$-nearest neighbors search. Furthermore, it does not require an explicit computation of the feature space, thus preserving one of the main advantages of prototype-based methods. Compared to the well-known SVM- $k$-NN local learning approach [2], MLNN also speeds up query processing: instead of learning a local classifier for each query, MLNN performs learning upwards, once and for all, and does not need to be run again or updated depending on queries (Fig. 2). Finally, the most significant advantage of MLNN lies in its ability to find out the most relevant prototypes for categorization, allowing to filter out the remaining less reliable examples. Experimentally, significant data reductions are observed with a simultaneous increase in categorization performances.

In Sec. 2 we present our MLNN approach, along with the statement of its theoretical properties. In order not to laden the paper's body, the proofsketches of the results have been postponed to an appendix. Then, Sec. 3 displays the behavior of MLNN on three standard databases of real-world image categorization. Finally, we conclude with some observations (Sec. 4).

\section{Method}

\subsection{Problem statement and notations}

In this paper, we address the task of multiclass image categorization. It consists in assigning an image to one of several predefined categories (or classes, or labels). Instead of splitting the multiclass problem in as many one-versus-all (binary) classification problems - a frequent approach in boosting [12] — we directly tackle the multiclass problem, following Zou et al [13]. For a given query image, we compute its classification score for all categories. While we basically use this vector for single-label prediction using the category with the maximum score, our algorithm can be straightforwardly extended to multilabel prediction and ranking [12]. We suppose given a set $\mathcal{S}$ of $m$ annotated images. Each image is a training example $(\boldsymbol{x}, \boldsymbol{y})$, where $\boldsymbol{x}$ is the image feature vector and $\boldsymbol{y}$ the class vector that specifies the category membership of the image. In particular, the sign of component $y_{c}$ gives the positive/negative membership of the example to class $c(c=1,2, \ldots, C)$. Inspired by the multiclass boosting analysis of Zou et al [13], we constrain the class vector to be symmetric, i.e.: $\sum_{c=1}^{C} y_{c}=0$, by setting: $y_{\tilde{c}}=1, y_{c \neq \tilde{c}}=-\frac{1}{C-1}$, where $\tilde{c}$ is the true image category. Furthermore, we denote by $K\left(\boldsymbol{x}_{\boldsymbol{i}}, \boldsymbol{x}_{\boldsymbol{j}}\right)$ a symmetric similarity kernel on the pair of examples $\boldsymbol{x}_{\boldsymbol{i}}, \boldsymbol{x}_{\boldsymbol{j}}$. 


\section{2 (Leveraged) $k$-Nearest Neighbors}

The vanilla $k$-NN rule is based on majority vote among the $k$-nearest neighbors in set $\mathcal{S}$, to predict the class of query $\boldsymbol{x}_{q}$. It can be defined as the following multiclass classifier $\boldsymbol{h}=\left\{h_{c}, c=1,2, \ldots, C\right\}$ :

$$
h_{c}\left(\boldsymbol{x}_{q}\right)=\frac{1}{k} \sum_{i \sim_{k} q}\left[y_{i c}>0\right]
$$

where $h_{c} \in[0,1]$ is the classification score for class $c, i \sim_{k} q$ denotes an example $\left(\boldsymbol{x}_{i}, \boldsymbol{y}_{i}\right)$ belonging to the $k$-nearest neighbors of $\boldsymbol{x}_{q}$ and square brackets denote the indicator function.

In this paper, we propose to generalize (1) to the following leveraged $k$-NN rule $\boldsymbol{h}^{\ell}=\left\{h_{c}^{\ell}\right\}$ :

$$
h_{c}^{\ell}\left(\boldsymbol{x}_{q}\right)=\sum_{j=1}^{T} \alpha_{j} K\left(\boldsymbol{x}_{q}, \boldsymbol{x}_{j}\right) y_{j c} \in \mathbb{R},
$$

where prediction $h_{c}^{\ell}$ takes values in all $\mathbb{R}$. In (2), we have introduced the three following elements to generalize (1):

- leveraging coefficients $\alpha_{j}$, that provide a weighted voting rule instead of uniform voting;

- kernel $K$, which takes into account "soft" (real-valued) similarities between query $\boldsymbol{x}_{q}$ and prototypes $\boldsymbol{x}_{j}$, instead of "hard" selection of the most similar ( $k$-NN) prototypes;

- size $T$ of the set of prototypes that are allowed to vote.

This last point is particularly interesting for computational purposes, as our classification rule actually involves only a (possibly sparse) subset of the training data as prototypes to be used at query time. Indeed, a prototype selection step is to be performed while training our classifier, in order to determine the most relevant subset of training data, i.e., the so-called prototypes, forming a set $\mathcal{P} \subseteq \mathcal{S}$ (Figure 2). The prototypes are selected during the training phase, which consists in fitting their coefficients $\alpha_{j}$, while removing the least relevant annotated data from $\mathcal{S}$.

\subsection{Multiclass surrogate risk minimization}

In order to fit our leveraged classification rule (2) onto training set $\mathcal{S}$, we focus on the minimization of a multiclass surrogate ${ }^{4}$ (exponential) risk:

$$
\varepsilon^{\exp }\left(\boldsymbol{h}^{\ell}, \mathcal{S}\right) \doteq \frac{1}{m} \sum_{i=1}^{m} \exp \left\{-\rho\left(\boldsymbol{h}^{\ell}, i\right)\right\},
$$

\footnotetext{
${ }^{4}$ We call surrogate a function that upperbounds the risk functional we should minimize, and thus can be used as a primer for its minimization.
} 
where:

$$
\rho\left(\boldsymbol{h}^{\ell}, i\right)=\frac{1}{C} \sum_{c=1}^{C} y_{i c} h_{c}^{\ell}\left(\boldsymbol{x}_{i}\right)
$$

is the multiclass edge of classifier $\boldsymbol{h}^{\ell}$ on training example $\boldsymbol{x}_{i}$. In particular, this edge averages over the $C$ classes the "goodness of fit" of classifier $\boldsymbol{h}^{\ell}$ on example $\left(\boldsymbol{x}_{i}, \boldsymbol{y}_{i}\right)$, thus being positive iff the prediction agrees with the example's annotation. Therefore, counting the number of negative edges enables to quantify the so-called empirical risk, i.e., the actual misclassification rate on the training data, as follows:

$$
\varepsilon^{0 / 1}\left(\boldsymbol{h}^{\ell}, \mathcal{S}\right) \doteq \frac{1}{m} \sum_{i=1}^{m}\left[\rho\left(\boldsymbol{h}^{\ell}, i\right)<0\right]
$$

Rather than directly tackling the problem of minimizing $\varepsilon^{0 / 1}$ - which is not differentiable and often computationally hard to minimize [14] — we concentrate on the optimization of surrogate (3), which is an upper bound of the empirical risk.

In order to solve this optimization, we propose a boosting-like procedure, i.e., an iterative strategy where the classification rule is updated by adding a new prototype $\left(\boldsymbol{x}_{j}, \boldsymbol{y}_{j}\right)$ (weak classifier) at each step $t(t=1,2, \ldots, T)$, thus updating the strong classifier (2) as follows:

$$
h_{c}^{(t)}\left(\boldsymbol{x}_{i}\right)=h_{c}^{(t-1)}\left(\boldsymbol{x}_{i}\right)+\delta_{j} K\left(\boldsymbol{x}_{i}, \boldsymbol{x}_{j}\right) y_{j c} .
$$

( $j$ is the index of the prototype chosen at iteration $t$.) Using (6) into (4), and then plugging it into (3), turns the problem of minimizing (3) to that finding $\delta_{j}$ with the following objective:

$$
\underset{\delta_{j}}{\arg \min } \sum_{i=1}^{m} w_{i} \cdot \exp \left\{-\delta_{j} \mathrm{r}_{i j}\right\} .
$$

In (7), we have defined $w_{i}$ as the weighting factor, depending on the past weak classifiers:

$$
w_{i}=\exp \left\{-\frac{1}{C} \sum_{c=1}^{C} y_{i c} h_{c}^{(t-1)}\left(\boldsymbol{x}_{i}\right)\right\},
$$

and $\mathrm{r}_{i j}$ as a pairwise term only depending on training data:

$$
\mathrm{r}_{i j}=K\left(\boldsymbol{x}_{i}, \boldsymbol{x}_{j}\right) \frac{1}{C} \sum_{c=1}^{C} y_{i c} y_{j c} .
$$

Finally, taking the derivative of (7), the global minimization of surrogate risk (3) amounts to fitting $\delta_{j}$ so as to solve the following equation:

$$
\sum_{i=1}^{m} w_{i} \mathrm{r}_{i j} \exp \left\{-\delta_{j} \mathrm{r}_{i j}\right\}=0 \text {. }
$$




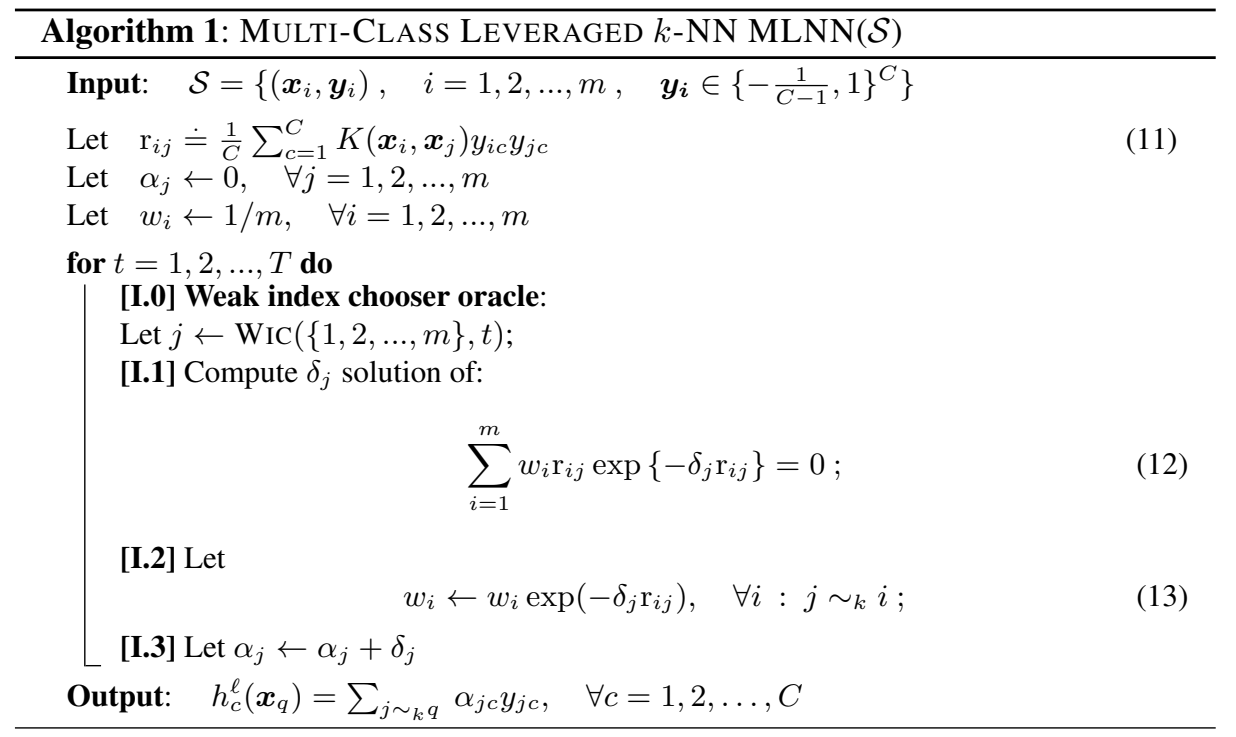

\subsection{MLNN: Multi-Class Leveraged $k$-NN rule}

Pseudocode of MLNN is shown in Alg. 1. The main ingredient to compute leveraging coefficients relies on the so-called edge matrix $\mathrm{R}$ with general entry $\mathrm{r}_{i, j}$ (Eq. 9). This term depends on the pairwise similarity between two training examples, as it is given by the kernel, as well as on the ground-truth annotations. Indeed, it combines a "labeling" term, which determines the sign, i.e., being positive iff labels of $i$ and $j$ agree, with a "geometric" term, which influences the magnitude, i.e., being larger when the two examples are closer to each other in the feature space.

We distinguish the following two cases, depending on which kernel is selected:

$k$-NN kernel In the most basic setting, i.e., when using $k$-NN kernel, term $K\left(\boldsymbol{x}_{i}, \boldsymbol{y}_{i}\right)$ behaves like an indicator function that only selects the $k$-NN of $i$. Therefore, in this case (9) simplifies to:

$$
\mathrm{r}_{i j} \doteq\left\{\begin{array}{cc}
\frac{1}{C} \sum_{c=1}^{C} y_{i c} y_{j c} & \text { if } j \sim_{k} i \\
0 & \text { otherwise }
\end{array}\right.
$$

and (10) has the following closed-form solution:

$$
\delta_{j} \leftarrow \frac{(C-1)^{2}}{C} \log \left(\frac{(C-1) w_{j}^{+}}{w_{j}^{-}}\right),
$$

with:

$$
w_{j}^{+}=\sum_{i: \mathrm{r}_{i j}>0} w_{i}, \quad w_{j}^{-}=\sum_{i: \mathrm{r}_{i j}<0} w_{i} .
$$


general kernel When using any kernel, entries of the edge matrix (Eq. 9) are realvalued and, in general, not sparse. Moreover, the equation (10) is transcendental, thus not admitting a closed-form solution. Hence, we compute the solution numerically, implementing a Newton's iterative method. This method gives the following approximation at step $k+1$, given the previous one at step $k$ :

$$
\delta^{(k+1)}=\delta^{k}+\frac{\sum_{i=1}^{m} w_{i} \mathrm{r}_{i j} \exp \left\{-\delta^{(k)} \mathrm{r}_{i j}\right\}}{\sum_{i=1}^{m} w_{i} \mathrm{r}_{i j}^{2} \exp \left\{-\delta^{(k)} \mathrm{r}_{i j}\right\}} .
$$

A critical setting for obtaining quick convergence of the solution is the initialization. Here, we propose to initialize the algorithm with the root of a linearized version of Eq. (10):

$$
\delta^{(0)}=\frac{\sum_{i=1}^{m} w_{i} \mathrm{r}_{i j}}{\sum_{i=1}^{m} w_{i} \mathrm{r}_{i j}^{2}} .
$$

A suitable choice for the kernel is the Radial Basis Function (RBF), which provides "smooth" pairwise similarities between feature points:

$$
K\left(\boldsymbol{x}_{i}, \boldsymbol{x}_{j}\right)=\exp \left\{-\frac{\left\|\boldsymbol{x}_{i}-\boldsymbol{x}_{j}\right\|^{2}}{2 \sigma^{2}}\right\},
$$

where parameter $\sigma$ may be either constant or adapted to the local sample density (e.g., one may set $\sigma=\rho_{k}\left(\boldsymbol{x}_{i}\right)$, where $\rho_{k}\left(\boldsymbol{x}_{i}\right)$ is the $k$-NN distance to $\boldsymbol{x}_{i}$, thus "enlarging" the window size where training data are sparser.) In particular, in the following experiments we use a Gaussian kernel that is truncated to the first $k$ nearest neighbors, thus providing a straightforward generalization of the $k$-NN kernel. In this case the edge matrix writes as follows:

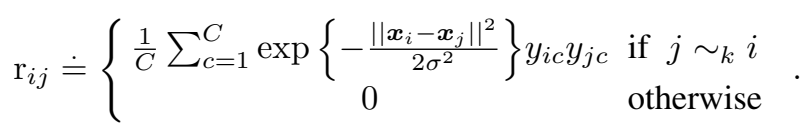

Another ingredient of MLNN is more common to boosting algorithms: MLNN operates on a set of weights $w_{i}(i=1,2, \ldots, m)$ defined over training data. These weights are repeatedly updated, such that those of mislabelled examples are increased, and vice-versa.

At each iteration $t$ of the algorithm, a weak index chooser oracle $\operatorname{WIC}(\{1,2, \ldots, m\}, t)$ determines index $j \in\{1,2, \ldots, m\}$ of the example to leverage (step I.0). Various choices are possible for this oracle. The simplest is perhaps to compute Eq. $(16,12)$ for all the training examples. $\delta_{j}$ in Eq. (12) can indeed be used to obtain a local measure of the class density [14], which is as better as $\delta_{j}$ gets large. This simple oracle thus picks $j$ maximizing $\delta_{j}$ :

$$
j \leftarrow \operatorname{WiC}(\{1,2, \ldots, m\}, t): \delta_{j}=\max _{j \in\{1,2, \ldots, m\}} \delta_{j}^{t} .
$$

This oracle allows an example to be chosen more than once, thus letting its leveraging coefficient $\alpha_{j}$ be updated several times (step I.3). It is known that, in order to be statistically consistent, some boosting algorithms require to be run for $T \ll m$ rounds [15]. 
Cast in the setting of MLNN, this constraint precisely supports prototype selection, as $T$ is an upperbound for the number of examples with non-zero leveraging coefficients.

MLNN shares the property with boosting algorithms of being resources-friendly: since computing the leveraging coefficients scales linearly with the number of neighbors, the time complexity bottleneck of MLNN does not rely on boosting, but rather on the complexity of $k$-NN search. Furthermore, notice that, when whichever $w_{j}^{+}$or $w_{j}^{-}$is zero, $\delta_{j}$ in (12) is not finite. There is a simple way to eliminate this drawback, inspired by [12]: we add $1 / m$ to both the numerator and the denominator of the fraction in the $\log$ term of (12). This smoothes out $\delta_{j}$, guaranteeing its finiteness without impairing convergence of MLNN.

In the following section, we provide formal details about the boosting analysis of MLNN.

\subsection{Properties of MLNN}

Two fundamental theorems hold for MLNN.

Theorem 1 MLNN converges with $T$ to $\boldsymbol{h}^{\ell}$ realizing the global minimum of the exponential risk (3).

MLNN is a specialization of a very general learning algorithm which keeps the same convergence guarantee when replacing the surrogate risk (3) by elements of a broad class of surrogates risk [15,14].

The second theorem provides a convergence rate for MLNN, which is based on a fundamental assumption on weak classifiers.

Theorem 2 Let $p_{j} \doteq w_{j}^{+} /\left(w_{j}^{+}+w_{j}^{-}\right)$. If the following weak index assumption (WIA) holds for $\tau \leq T$ steps in MLNN:

(WIA) There exist some $\gamma>0$ and $\eta>0$ such that the following two inequalities hold for index $j$ returned by $\operatorname{WIC}(\{1,2, \ldots, m\}, t)$ :

$$
\begin{aligned}
\left|p_{j}-1 / C\right| & \geq \gamma, \\
\left(w_{j}^{+}+w_{j}^{-}\right) /\|\boldsymbol{w}\|_{1} & \geq \eta .
\end{aligned}
$$

Then: $\varepsilon^{0 / 1}\left(\boldsymbol{h}^{\ell}, \mathcal{S}\right) \leq \exp \left(-\frac{C}{C-1} \eta \gamma^{2} \tau\right)$.

(Proofsketch in appendix) Ineq. (22) is the usual weak learning assumption, used to analyze classical boosting algorithms [16,12], when considering examples as weak classifiers. A weak coverage assumption (23) is needed as well, because insufficient coverage of the reciprocal neighbors could easily wipe out the surrogate risk reduction due to a large $\gamma$ in (22). In the framework of $k$-NN classification, choosing $k$ not too small is enough for the WIA to be met for a large number of boosting rounds $\tau$, thus determining a potential harsh decrease of $\varepsilon^{\exp }\left(\boldsymbol{h}^{\ell}, \mathcal{S}\right)$. This is important, as a big difference with classical boosting algorithms (e.g. AdaBoost [16]) is that oracle $\operatorname{WIC}(.,$. has only access to $m$ different weak classifiers, i.e., one per example. Finally, the bound in Theorem 2 shows that classification (22) may be more important than coverage (23) for nearest neighbors. 


\section{Experiments}

In this section, we present experimental results of MLNN with different kernel settings and comparison with both $k$-NN and ITML [6], which is a state-of-the-art metric learning algorithm. In particular, our experiments aim at evaluating the effect of UNN sparse prototype selection on the classification accuracy. For this purpose, we measured the classification performances when varying the number of prototypes retained at test time. In MLNN, prototype selection is carried out by setting $T<m$, which corresponds to retaining at most $T$ relevant prototypes. When running the other methods, we carried out random prototype selection and averaged the results over a number of iterations.

\subsection{Scene categorization}

We validated our MLNN algorithm on three well-known image categorization databases.

8-cat: firstly proposed by [1], includes 2,688 color images grouped into eight categories: 360 coast, 328 forest, 374 mountain, 410 open country, 260 highway, 308 inside of cities, 356 tall buildings, and 292 street (Fig. 1).

13-cat: adds five more categories of gray-scale images to the 8-cat database [17]: 241 suburb residence, 174 bedroom, 151 kitchen, 289 living room, and 216 office.

15-cat: includes 13-cat database plus two more categories (gray-scale images) [18]: 315 store, and 311 industrial.

In the following section we report results obtained by splitting each database in two distinct subsets, one for training, the other for test. We always used about 2,000 randomly selected training images. Namely, 250 images per category were selected from the 8-cat database, 150 from the other two datasets. The remaining images were used for testing. In our experiments, we mostly concentrated on evaluating the tradeoff between classification accuracy and computational time, as provided by selecting a sparse prototype dataset from the training data. In particular, fixing the number of prototypes amounts to fixing the computational cost of classification, as this latter only depends on the cost of $k$-NN search on the prototype set. (So as for $k$-NN, a random sample of the training set was selected and results were averaged over a number of random sampling realizations.) All the results we present were obtained with $k=11$ and pre-processing Gist features [1] with PCA down to dimension $d=128$.

We compared different implementations of our MLNN algorithm. In particular, we tested:

- MLNN with the basic setting, i.e., the uniform $k$-NN kernel of Eq. (14);

- WMLNN, i.e., MLNN with fixed-size Gaussian kernel (19) (with $\sigma=0.25$ );

- AdaWMLNN, i.e., MLNN with adaptive-size Gaussian kernel (19) (with $\sigma=$ $\sqrt{2} \rho_{k}\left(\boldsymbol{x}_{i}\right), \rho_{k}\left(\boldsymbol{x}_{i}\right)$ being the $k$-NN distance from example $\left.\boldsymbol{x}_{i}\right)$;

- MLNN "one-versus-all”, i.e. Alg. 1 with $C=2$ applied to each category independently (considering examples in the current category as "positives", the remaining ones as "negatives"). 
Furthermore, we compared our method with different $k$-NN-based classification methods, which either rely on metric learning or not. Namely, we tested:

- classic non-parametric $k$-NN voting;

- weighted $k$-NN (W $k-\mathrm{NN}$ ) voting with Gaussian weights, as proposed by Philbin et al. [19]; we used (19) with $\sigma=1$ as a weighting factor;

- $k$-NN voting combined with ITML metric learning [6].

We tested all these methods for a fixed number of prototypes, i.e., for a fixed computational cost of classification. In particular, a random sample of the training set was selected and results were averaged over a number of random sampling realizations.

Finally, we integrated the ITML method with MLNN in order to provide a unique method for addressing simultaneously both the choice of the metric distance and the rejection of "noisy" examples, which are the two fundamental issues of $k$-NN classification.

\subsection{Categorization results}

The categorization test consists in assigning each test image to one of the predefined categories. We measured the overall performance rate as the mean Average Precision (mAP), which is the average of the classification rates for each category.

In Fig. 3(a) we compare the results of MLNN with the abovementioned settings. Interestingly, these results show the significant improvement provided by using a "smooth" kernel for learning the prototypes. Namely, the adaptive-size kernel provides the best performances. Furthermore, the gap over the basic MLNN is more consistent when retaining less prototypes, as AdaMLNN enables a finer class density estimation even with very sparse examples (see, for instance, the performance gap of 7\% between MLNN and AdaWMLNN for $T=200$ ). Furthermore, notice that the multiclass version of our algorithm outperforms the one-versus-all implementation (gap between 1\% and 3\%). Hence, our multiclass MLNN not only is much less computationally expensive than one-versus-all MLNN, as it avoids to run the boosting procedure $C$ times independently, but also provides better classification accuracy.

On the same 8-cat database we compared AdaWMLNN to $k$-NN voting with or without metric learning (Fig. 3(b)). First of all, we notice that our AdaWMLNN method significantly outperforms $k$-NN and $\mathrm{W} k$-NN, i.e., non-learned voting rules (up to $6 \%$ improvement). Then, performances of our method are overall comparable to those of ITML, being slightly inferior to them, but the computational cost of MLNN is considerably lower than that of metric learning. Finally, our results show that, when combined with a metric learning strategy, MLNN is able to significantly outperform all the other classification methods, thus enabling a significant accuracy improvement over the state-of-the-art (up to 3\% when retaining few prototypes, see for example performance at $T=200)$.

In Fig. 4 we focus on a more extensive comparison between regular MLNN and classic $k$-NN on the 13-cat and 15-cat datasets. Here, we report the mean Average Precision as a function of the number of prototypes per category. (Since this number varies from category to category, we report the average number of prototypes over all 


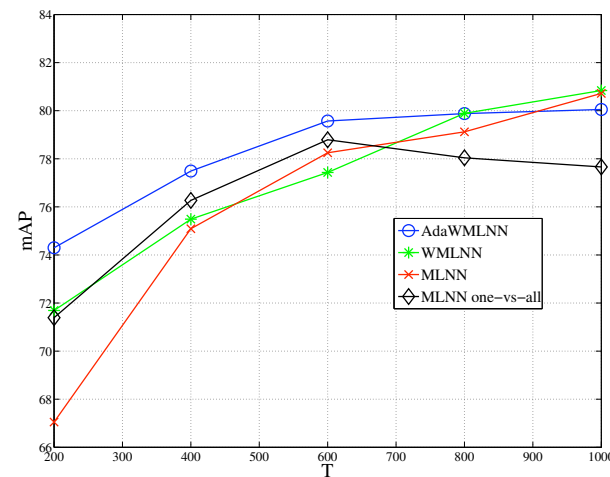

(a)

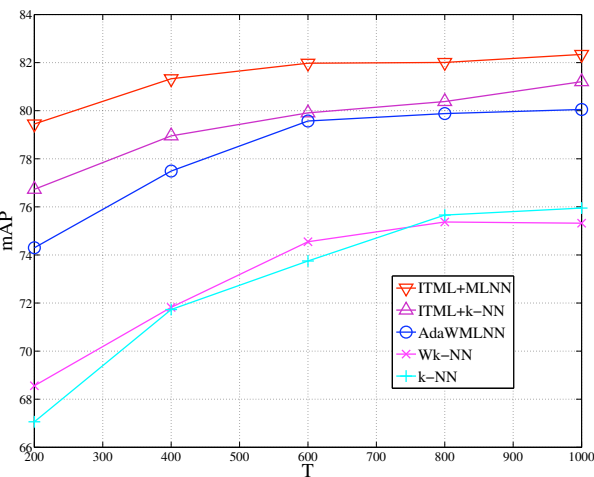

(b)

Fig. 3. Experimental results of categorization on 8-cat database in terms of mAP as a function of the number of prototypes, for $k=11$ and Gist descriptors of dimension $d=128$ (after PCA). (a) Comparison between 3 different implementations of MLNN and one-versus-all MLNN. (b) Comparison between MLNN with adaptive Gaussian kernel (AdaWMLNN), $k$-NN, weighted $k$-NN and MLNN one-versus-all (UNN).

categories.) Notice that the gap between the two methods is most significant when retaining less than half prototypes, namely $6 \%$ improvement with 80 prototypes on 8 -cat database, $7 \%$ with only 60 prototypes for both 13-cat and 15-cat. Besides considerably improving precision over $k$-NN, we also drastically reduce the computational complexity of classification, which deals with finding nearest neighbors on a sparse dataset (gain up to a factor 4 when discarding half prototypes).

\section{Conclusion}

In this paper, we have proposed a novel boosting algorithm, MLNN, which learns a leveraged $k$-NN rule following the minimization of a multiclass surrogate (exponential) risk. This rule generalizes $k$-NN to weighted voting. Under mild learning and coverage assumptions, MLNN convergence is proven to be exponentially fast. Experiments on benchmark image categorization databases display that MLNN is significantly more accurate than $k$-NN (up to $6 \%$ ), achieving very significant improvement on image databases with only few thousand images. Since the number of weak hypotheses available for boosting is in the order of the number of images, improvements may rapidly become dramatic as databases get larger. MLNN also provides us with a very simple and efficient prototype selection method reducing the cost of searching for neighbors at classification time. MLNN precision is comparable with that of a stateof-the-art metric learning method [6]. Since MLNN is still fully compatible with any underlying distortion and data structure for $k$-NN search, it also take advantage from learning a metric distance, thus simultaneously solving both major issues of $k$-NN voting: selection of a suitable metric distance and rejection of "noisy" prototypes. Last but 

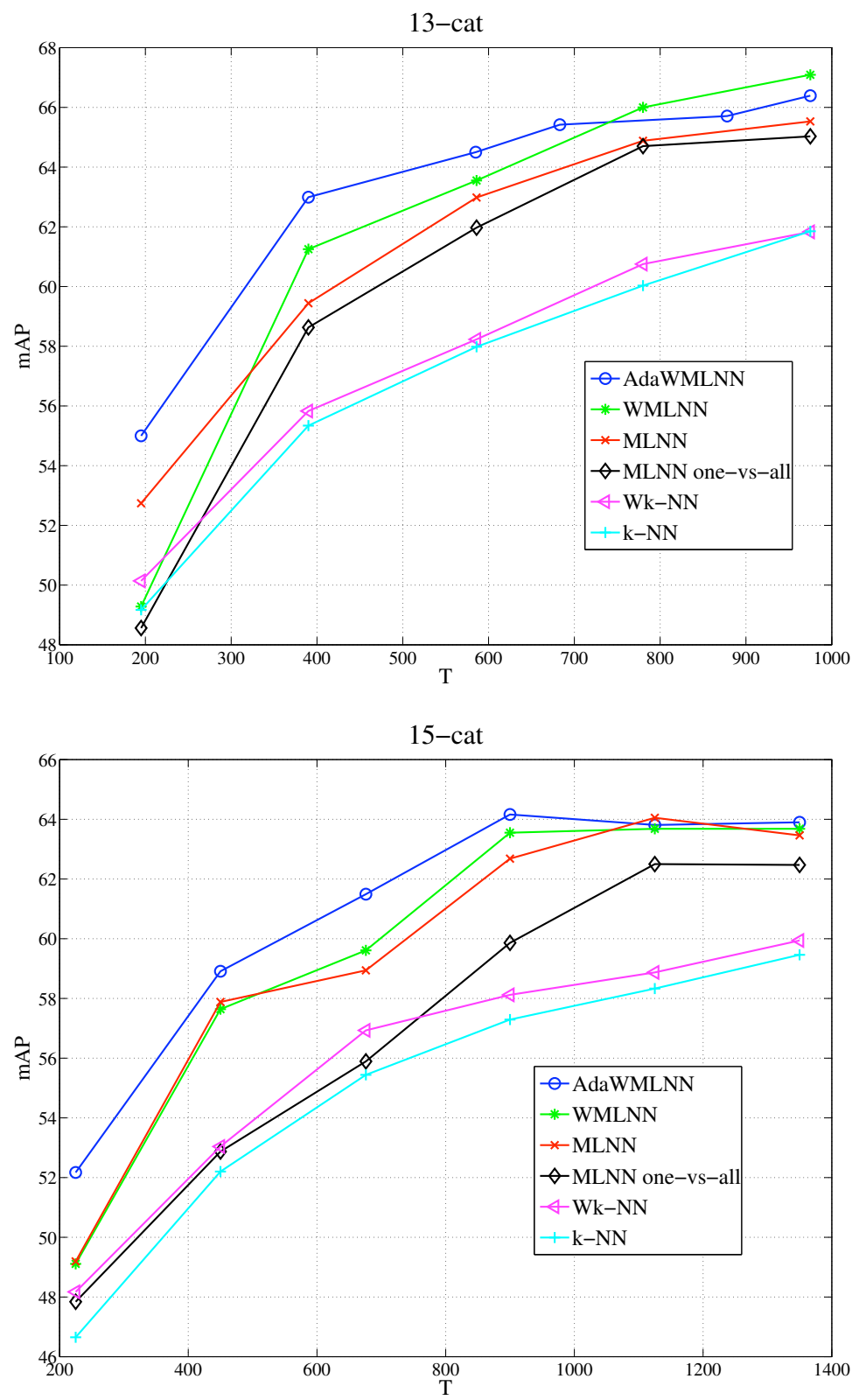

Fig. 4. Performance of MLNN with different settings (see the paper for details) compared to $k-\mathrm{NN}$ and weighted $k$-NN as a function of the number of prototypes per class for 13-cat (4(a)) and 15-cat (4(b)) datasets. 
not least, MLNN is simple and modular enough, so that it can be easily extended to work on global image descriptors (like Bags of Features, Fisher Kernels ...)

\section{References}

1. Oliva, A., Torralba, A.: Modeling the shape of the scene: A holistic representation of the spatial envelope. Int. J. of Comp. Vision 42 (2001) 145-175 2, 9

2. Zhang, H., Berg, A.C., Maire, M., Malik, J.: Svm-knn: Discriminative nearest neighbor classification for visual category recognition. In: CVPR'06. (2006) 2126-2136 2, 3

3. Boiman, O., Shechtman, E., Irani, M.: In defense of nearest-neighbor based image classification. In: CVPR'08. (2008) 1-8 2

4. Hastie, T., Tibshirani, R.: Discriminant adaptive nearest neighbor classification. IEEE Trans. PAMI 18 (1996) 607-616 2

5. Paredes, R.: Learning weighted metrics to minimize nearest-neighbor classification error. IEEE Trans. PAMI 28 (2006) 1100-1110 Member-Vidal, Enrique. 2

6. Davis, J.V., Kulis, B., Jain, P., Sra, S., Dhillon, I.S.: Information-theoretic metric learning. In: ICML'07. (2007) 209-216 2, 9, 10, 11

7. Brighton, H., Mellish, C.: Advances in instance selection for instance-based learning algorithms. Data Mining and Knowledge Disc. 6 (2002) 153-172 2

8. Zuo, W., Zhang, D., Wang, K.: On kernel difference-weighted k-nearest neighbor classification. Pattern Anal. Appl. 11 (2008) 247-257 2

9. Holmes, C.C., Adams, N.M.: Likelihood inference in nearest-neighbour classification models. Biometrika 90 (2003) 99-112 2

10. Marin, J.M., Robert, C.P., Titterington, D.M.: A Bayesian reassessment of nearest-neighbor classification. J. of the Am. Stat. Assoc. (2009) 2

11. Athitsos, V., Sclaroff, S.: Boosting nearest neighbor classi.ers for multiclass recognition. In: CVPR '05: Proceedings of the 2005 IEEE Computer Society Conference on Computer Vision and Pattern Recognition (CVPR'05) - Workshops. (2005) 453

12. Schapire, R.E., Singer, Y.: Improved boosting algorithms using confidence-rated predictions. Machine Learning 37 (1999) 297-336 3, 8

13. Zou, H., Zhu, J., Hastie, T.: New multicategory boosting algorithms based on multicategory fisher-consistent losses. Annals of Applied Statistics 2(4) (2008) 1290-1306 3

14. Nock, R., Nielsen, F.: Bregman divergences and surrogates for learning. IEEE Trans. PAMI 31 (2009) 2048-2059 5, 7, 8

15. Bartlett, P., Jordan, M., McAuliffe, J.D.: Convexity, classification, and risk bounds. J. of the Am. Stat. Assoc. 101 (2006) 138-156 7, 8

16. Freund, Y., Schapire, R.E.: A Decision-Theoretic generalization of on-line learning and an application to Boosting. Journal of Comp. Syst. Sci. 55 (1997) 119-139 8

17. Fei-Fei, L., Perona, P.: A bayesian hierarchical model for learning natural scene categories. CVPR (2005) 524-5319

18. Lazebnik, S., Schmid, C., Ponce, J.: Beyond bags of features: Spatial pyramid matching for recognizing natural scene categories. In: CVPR. (2006) 2169-2178 9

19. Philbin, J., Chum, O., Isard, M., Sivic, J., Zisserman, A.: Lost in quantization: Improving particular object retrieval in large scale image databases. In: Proceedings of the IEEE Conference on Computer Vision and Pattern Recognition. (2008) 10 


\section{Appendix}

Proofsketch of Theorem 2 Without loss of generality and to simplify notations, assume that $j=t$ in Alg. 1, and denote $\boldsymbol{w}_{j}$ the weight vector on which we compute (12) - thus, the weight update in (13) gives $\boldsymbol{w}_{t+1}$, and the first weight vector is $\boldsymbol{w}_{1}$. Let us denote $Z_{t} \doteq\left\|\boldsymbol{w}_{\boldsymbol{t}+\mathbf{1}}\right\|_{1}$ the normalization coefficient for weights, and $\tilde{w}_{(t+1) i} \doteq w_{t i} / Z_{t}$ the normalized weight of example $\left(\boldsymbol{x}_{i}, \boldsymbol{y}_{i}\right)$ in the $(t+1)^{\text {th }}$ weight vector. Few derivations lead:

$$
\varepsilon^{\exp }\left(\boldsymbol{h}^{\ell}, \mathcal{S}\right)=\prod_{t=1}^{T} Z_{t}
$$

We now compute an upperbound for $Z_{t}$, removing the $t$ index for readability. For this objective, we extend notations (16) to the normalized tilda notation above, and let $\varrho \doteq$ $\min _{i, t: K\left(\boldsymbol{x}_{i}, \boldsymbol{x}_{t}\right)>0} K\left(\boldsymbol{x}_{i}, \boldsymbol{x}_{t}\right)$ and $\bar{\varrho} \doteq \max _{i, t} K\left(\boldsymbol{x}_{i} \boldsymbol{x}_{t}\right)$. Due to the lack of place, we make the proof in the simpler case where $\underline{\varrho}=\bar{\varrho}=1$. We obtain:

$$
\begin{aligned}
Z=\sum_{i=1}^{m} \tilde{w}_{i} \exp \left(-\delta_{j} \mathrm{r}_{i j}\right) \leq & \tilde{w}_{j}^{+} \exp \left(-\underline{\varrho} \frac{C-1}{C} \log \left(\frac{(C-1) w_{j}^{+}}{w_{j}^{-}}\right)\right)+ \\
& +\left(1-\tilde{w}_{j}^{-}-\tilde{w}_{j}^{+}\right)+\tilde{w}_{j}^{-} \exp \left(\frac{\bar{\varrho}}{C} \log \left(\frac{(C-1) w_{j}^{+}}{w_{j}^{-}}\right)\right)= \\
& =1-\tilde{w}_{j}^{-}-\tilde{w}_{j}^{+}+\frac{C}{C-1}\left((C-1) \tilde{w}_{j}^{+}\right)^{\frac{1}{C}}\left(\tilde{w}_{j}^{-}\right)^{1-\frac{1}{C}}= \\
& =1-\left(\tilde{w}_{j}^{-}+\tilde{w}_{j}^{+}\right)\left[1-\frac{C\left((C-1) \tilde{\tilde{w}}_{j}^{+}\right)^{\frac{1}{C}}\left(\tilde{\tilde{w}}_{j}^{-}\right)^{1-\frac{1}{C}}}{C-1}\right]
\end{aligned}
$$

where we have used the shorthands $\tilde{\tilde{w}}_{j}^{+} \doteq w_{j}^{+} /\left(w_{j}^{+}+w_{j}^{-}\right)$and $\tilde{\tilde{w}}_{j}^{-} \doteq w_{j}^{-} /\left(w_{j}^{-}+w_{j}^{+}\right)$. Using the WIA (23) and the fact that $1-x \leq \exp (-x)$, we obtain from (25):

$$
\begin{aligned}
Z & \leq \exp \left[-\eta\left(1-f\left(\tilde{\tilde{w}}_{j}^{+}\right)\right)\right], \\
f(x) & \doteq \frac{C}{C-1}((C-1) x)^{\frac{1}{C}}(1-x)^{1-\frac{1}{C}}, x \in[0,1] .
\end{aligned}
$$

$f(x)$ is concave on $[0,1]$ and admits a maximum in $x=1 / C$; Assuming the WIA (22), we get $\left|\tilde{\tilde{w}}_{j}^{+}-1 / C\right| \geq \gamma$. If $x \leq 1 / C-\gamma$, then $f(x) \leq g_{-}(\gamma)$, and if $x \geq 1 / C+\gamma$, then $f(x) \leq g_{+}(\gamma)$, with:

$$
\begin{aligned}
& g_{-}(\gamma) \doteq(1-C \gamma)^{\frac{1}{C}}\left(1+\frac{C}{C-1} \gamma\right)^{1-\frac{1}{C}} \\
& g_{+}(\gamma) \doteq(1+C \gamma)^{\frac{1}{C}}\left(1-\frac{C}{C-1} \gamma\right)^{1-\frac{1}{C}}
\end{aligned}
$$

But it can be shown that both $g_{-}(\gamma)$ and $g_{+}(\gamma)$ can be upperbounded by $g(\gamma)=1-$ $C \gamma^{2} /(C-1), \forall C \geq 2, \forall \gamma \in[0,1]$. Plugging the bound in (26), we obtain:

$$
Z \leq \exp \left[-\eta\left(1-g\left(\tilde{\tilde{w}}_{j}^{+}\right)\right)\right]=\exp \left[-\frac{C}{C-1} \eta \gamma^{2}\right] \text {. }
$$

Finally, $Z \leq 1$ because $0 \leq f_{C}(x) \leq 1$ for any $x \in[0,1]$, and (24) yields $\varepsilon^{\exp }\left(\boldsymbol{h}^{\ell}, \mathcal{S}\right) \leq$ $\exp \left(-C \eta \gamma^{2} \tau /(C-1)\right)$. Using the fact that $\varepsilon^{0 / 1}\left(\boldsymbol{h}^{\ell}, \mathcal{S}\right) \leq \varepsilon^{\exp }\left(\boldsymbol{h}^{\ell}, \mathcal{S}\right)$ yields the proof of Theorem 2. 